\title{
Elevated serum IL-8 levels are associated with disease activity in idiopathic intermediate uveitis
}

\author{
Anne-Marie Klok, Leny Luyendijk, Michel J W Zaal, Aniki Rothova, C Erik Hack, \\ Aize Kijlstra
}

Department of

Ophthalmo-Immunology,

Netherlands

Ophthalmic Research

Institute, Amsterdam

A $M$ Klok

L Luyendijk

A Kijlstra

Department of

Ophthalmology, Free

University,

Amsterdam

A M Klok

M J W Zaal

Department of

Ophthalmology

University of Utrecht,

Utrecht

A Rothova

Department of

Ophthalmology,

University of

Amsterdam

A Kijlstra

Central Laboratory of the Netherlands Red

Cross Blood

Transfusion Service,

Amsterdam and

Laboratory for

Experimental and

Clinical Immunology,

University of

Amsterdam

C E Hack

Correspondence to:

Anne-Marie Klok, MD,

Department of

Ophthalmo-Immunology,

Netherlands Ophthalmic

Research Institute,

Amsterdam, PO Box 12141,

1100 AC Amsterdam,

Netherlands.

Accepted for publication

26 February 1998
Aim-To find a laboratory indicator for systemic involvement in intermediate uveitis.

Methods-Interleukin 8 (IL-8) and C reactive protein (CRP) serum levels were measured in patients with idiopathic intermediate uveitis $(n=61)$, uveitis controls $(n=143)$, and normal controls $(n=29)$. The records of those with intermediate uveitis were reviewed with the emphasis on disease activity and severity as characterised by the presence of cystoid macular oedema, vitreous exudates or snowbank formation, papillitis, and periphlebitis.

Results-Increased serum IL-8 ( $\geqslant 20$ pg/ ml) was found in 27 out of 61 patients with intermediate uveitis $(p<0.01), 12$ of 27 patients with sarcoid uveitis $(p<0.05)$, in 19 of 30 patients with HLA-B27 associated acute anterior uveitis $(p<0.05)$, and in five of 29 healthy controls. Raised IL-8 levels in intermediate uveitis were significantly associated with active disease $(p<0.001)$ and the presence of vitreous exudates $(\mathbf{p}<0.001)$, papillitis, and periphlebitis $(p<0.01)$. Elevated CRP levels were found in 12 of the 143 uveitis controls but in none of the intermediate uveitis patients or normal controls. During follow up an associated systemic disease was more frequently noticed in patients with an elevated serum IL-8 at entry into the study. Conclusions-Elevated IL-8 serum levels were found in patients with active intermediate uveitis of unknown origin. An elevated IL-8 level seems to predispose the patient to a later development of associated systemic disease.

(Br f Ophthalmol 1998;82:871-874)

Intermediate uveitis is an inflammation in the region of the posterior ciliary body, anterior retina, anterior choroid, and vitreous. It is characterised by cells and debris in the vitreous, snowbank formation along the pars plana, varying degrees of periphlebitis, and a relatively quiet anterior chamber. It generally occurs bilaterally and usually affects young patients although it can also be seen later in life. The course of intermediate uveitis is variable, ranging from a self limited process to chronic disease with remissions and exacerbations. It can result in severe complications including cystoid macular oedema (CMO), epiretinal membrane formation, cataract, neovascularisation, vitreous haemorrhage, and retinal detachment. ${ }^{1-4}$
It is not clear how many disease entities are included in the category of intermediate uveitis. The aetiology of intermediate uveitis is still unknown although some patients are subsequently found to have other underlying systemic disease responsible for the ocular process. The main systemic diseases associated with intermediate uveitis are sarcoidosis and multiple sclerosis. ${ }^{5-7}$ To establish these latter associations a number of well defined clinical and laboratory variables are currently available. When the patient presents with eye disease the outcome of these tests may however not yet be evident. Furthermore, it is possible that other as yet unknown systemic disorders may also be involved in intermediate uveitis. One way to find an indication for systemic involvement is to assess acute phase reactants. ${ }^{8}$ In this study we therefore measured the serum concentration of $\mathrm{C}$ reactive protein (CRP): its level in the serum increases rapidly from a normal level of $0.8 \mathrm{mg} / 1$ to as much as several hundred milligrams per litre in response to most forms of tissue injury, inflammation, or infection. ${ }^{9} 10$

To find another indication for the presence of a systemic disease in intermediate uveitis we also measured the presence of the chemokine interleukin 8 (IL-8). IL-8 is one of the main chemoattractants for neutrophils and can also activate the neutrophils. ${ }^{11}$ Elevated levels of IL- 8 have been reported in several systemic inflammatory diseases such as sepsis and sarcoidosis. ${ }^{12} 13$ Tissue levels of IL- 8 and circulating levels of IL-8 antibodies appeared to be elevated in active ulcerative colitis. ${ }^{14}$ Raised serum IL-8 has also been found in the serum of patients with eye diseases wich are related to a systemic disease including proliferative diabetic retinopathy and HLA-B27 associated anterior uveitis (S Sprenkels, personal communication). In an earlier study we reported raised serum IL-8 levels in 11 of 20 samples from patients with intermediate uveitis. ${ }^{15}$ In this study we measured serum IL-8 in a larger group of intermediate uveitis patients and extended our earlier findings by investigating a possible relation with disease activity.

\section{Patients and methods}

We studied 61 patients with intermediate uveitis who visited the ophthalmology department of the Academic Hospital of the Free University of Amsterdam and the Academic Hospital of Utrecht in the period between 1981 and 1996. For the uveitis screening, at the first visit to one of our clinics blood samples were obtained by venepuncture, allowed to clot, and 
Table 1 Elevated IL-8 levels (>20 pg/ml) in serum of uveitis patients

\begin{tabular}{llcll}
\hline Diagnosis & No/total & $\begin{array}{c}\text { Median } \\
(p g / m l)\end{array}$ & Range $(p g / m l)$ & $p$ Value \\
\hline Intermediate uveitis & $27 / 61$ & 700 & $20-5823$ & $<0.01$ \\
Sarcoidosis & $12 / 27$ & 1600 & $26-6197$ & $<0.05$ \\
HLA-B27 AAU & $19 / 30$ & 2914 & $22-6051$ & $<0.05$ \\
Toxoplasmosis & $5 / 31$ & 1152 & $40-3040$ & \\
Behçet's syndrome & $5 / 25$ & 188 & $30-715$ & \\
Fuchs' cyclitis & $9 / 30$ & 1933 & $83-5483$ & \\
Controls & $5 / 29$ & 213 & $24-833$ & \\
\hline
\end{tabular}

AAU $=$ associated anterior uveitis.

${ }^{\star}$ Median levels of serum containing IL-8 levels above $20 \mathrm{pg} / \mathrm{ml}$.

Statistical analysis was performed using the Mann-Whitney U test whereby uveitis groups were compared with controls.

centrifuged for 10 minutes at $1500 \mathrm{~g}$ to obtain the serum. Serum was stored at $-20^{\circ} \mathrm{C}$.

The charts of these 61 patients with intermediate uveitis were reviewed. For inclusion in this study, the intraocular inflammation at time of diagnosis, had to be characterised predominantly by cellular infiltration in the vitreous, pars plana, or peripheral retina. The presence of snowbank formation was not obligatory. At the time of entry, the patients' personal and family histories were reviewed. Patients were questioned for any history of arthralgias, skin rash, gastrointestinal symptoms, or neurological symptoms. Complete ophthalmic examination was performed in all cases. All patients underwent a complete examination for uveitis. ${ }^{1}$ All results were within normal limits. The patients with known associated systemic disease such as multiple sclerosis or sarcoidosis at entry in the study were not included. At the time of venepuncture the following data were scored: the presence of CMO (confirmed with fluorescein angiography), vitreous exudates or snowbank formation, papillitis, and periphlebitis. Disease activity was divided in active or smouldering. Active disease was defined as an exacerbation of the disease with marked inflammatory reaction with cells and debris in the vitreous (Hogan and Kimura, grade $2-4)^{1}$ (at time of venepuncture). In smouldering disease there was no exacerbation but a minimal to moderate cellular infiltration was still present. Mean follow up after blood collection was 6.7 years with a range of $1-15$ years.

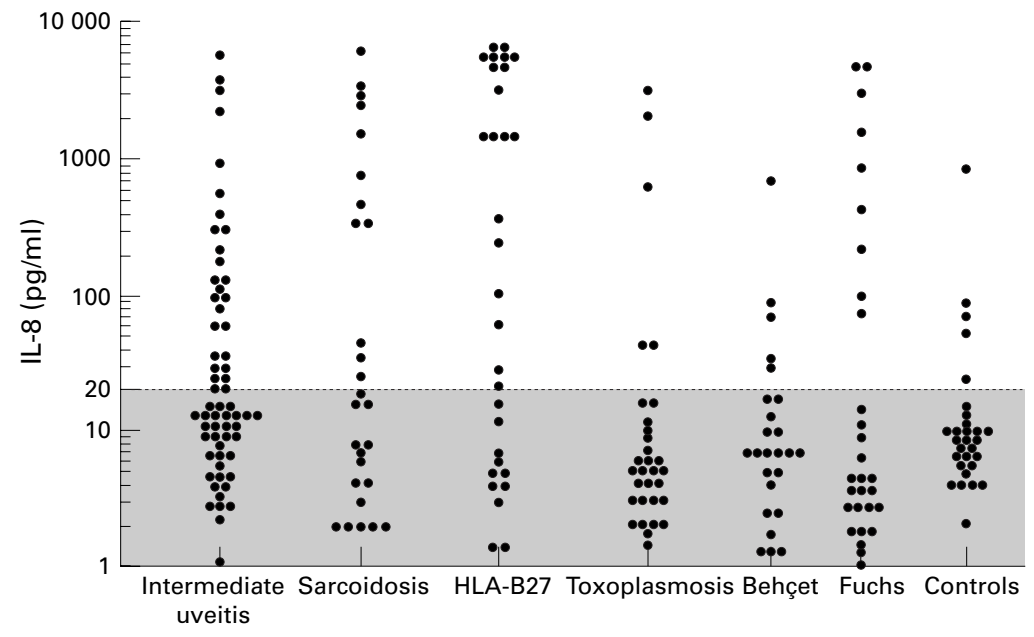

Figure 1 IL-8 levels in serum from intermediate uveitis patients, uveitis controls, and normal controls. The broken line represents the detection limit of the assay.
Serum samples of 143 uveitis controls with active disease (27 patients with sarcoidosis, 30 HLA-B27 acute anterior uveitis, 31 toxoplasma chorioretinitis, 25 Behçet uveitis, and 30 Fuchs' heterochromic cyclitis) and 29 healthy controls were obtained and processed as described above. These sera were collected in the same time as the intermediate uveitis group and stored under similar conditions. A diagnosis of uveitis was made according to the criteria of the International Uveitis Study Group. ${ }^{16}$

Serum levels of CRP were assessed by using an immunodiffusion assay according to the manufacturer's recommendations (LCPartigen; Behring, Marburg, Germany). CRP levels of $<10 \mathrm{mg} / 1$ were considered normal. ${ }^{17}$

IL-8 was measured with an enzyme linked immunoassay (ELISA) in which a monoclonal anti-human IL-8 antibody and a polyclonal anti-human IL-8 were used (Central Laboratory of the Netherlands Red Cross Blood Transfusion Service (CLB), Amsterdam). The preparation of these antibodies and the development of the IL-8 ELISA have been described elsewhere. ${ }^{12}$ ELISA plates were coated overnight with a monoclonal antibody against IL-8. After washing, $100 \mu \mathrm{l}$ of the sample dilutions (1:5 in a high performance ELISA buffer, CLB) were added in each well and incubated for 1 hour. After washing, the plates were incubated with $100 \mu \mathrm{l}$ biotinylated polyclonal sheep anti-IL-8 antibody (CLB) for 1 hour. Then the plates were developed with streptavidinhorseradish peroxidase followed by tetramethylbenzidine as a substrate. Purified human recombinant IL-8 (rIL-8) obtained by transfecting Escherichia coli DH5 with the plasmid pMBL11 (British Biotechnology Ltd, Oxford) was used as a standard and results were expressed in picograms per millilitre. ${ }^{18}$ The concentration of IL-8 in samples was calculated by comparing the absorption of samples with that of serial dilutions of human rIL-8. The detection limit of the assay was $20 \mathrm{pg} / \mathrm{ml}$.

Statistical analysis of the data was performed using Mann-Whitney $U$ test and $\chi^{2}$ analysis.

\section{Results}

CRP levels were within normal limits in all patients with intermediate uveitis. Elevated CRP levels were found in 12 of the 143 uveitis controls (in two of 27 sarcoidosis patients, in four of 30 HLA-B27 acute anterior uveitis, in four of 25 patients with Behçet's syndrome, and in two of 30 patients with Fuchs' heterochromic cyclitis) and in none of the normal controls.

IL- 8 was detectable in the serum of 27 of the 61 patients with intermediate uveitis (Table 1, Fig 1). Circulating IL-8 was also found in five of the 29 normal controls and in 31 of the 143 uveitis controls. IL-8 in the serum of intermediate uveitis patients was significantly higher than in the normal controls $(\mathrm{p}<0.01)$. The uveitis controls were subdivided in specific entities. Elevated IL-8 levels were found in 12 of 27 serum samples of sarcoidosis patients, in 19 of 30 with HLA-B27 acute anterior uveitis, in five of 31 with toxoplasmosis, in five of 25 
Table $2 \quad I L-8$ and clinical aspects of intermediate uveitis

\begin{tabular}{|c|c|c|c|}
\hline & $\begin{array}{l}\text { IL-8 pos* } \\
(27)\end{array}$ & $\begin{array}{l}\text { IL-8 neg } \\
\text { (34) }\end{array}$ & $p$ Value \\
\hline $\mathrm{CMO}$ & 10 & 13 & \\
\hline Vitreous exudates & 24 & 14 & $<0.001$ \\
\hline Papillitis & 8 & 0 & \\
\hline Periphlebitis & 11 & 3 & $<0.01$ \\
\hline
\end{tabular}

*IL-8 level above $20 \mathrm{pg} / \mathrm{ml}$.

Statistical analysis was performed using the $\chi^{2}$ test.

with Behçet's syndrome, and in nine of 30 patients with Fuchs' heterochromic cyclitis. IL-8 was significantly higher in patients with sarcoidosis $(p<0.05)$ and in patients with a HLA-B27 acute anterior uveitis $(\mathrm{p}<0.05)$ when compared with normal controls.

To investigate the relation between serum IL-8 levels and disease activity the patients with intermediate uveitis were analysed for the presence of CMO, vitreous exudates and snowbank formation, papillitis, and periphlebitis (Table 2). CMO was present in $10 \mathrm{IL}-8$ positive patients and in 13 IL- 8 negative; vitreous exudates were seen in 24 IL- 8 positive patients and in $14 \mathrm{IL}-8$ negative; periphlebitis was seen in 11 positive patients and in three negative patients. Papillitis was found in only eight IL-8 positive but in none of the negative patients. Vitreous exudates and periphlebitis were seen more often in IL- 8 positive patients compared with IL- 8 negative patients ( $p$ $<0.001 ; \mathrm{p}<0.01$, respectively). Active disease was observed more often in patients with high circulating IL-8 $(\mathrm{p}<0.001)$ (Table 3).

There was no evidence that IL- 8 was related to medical treatment (data not shown). Most intermediate uveitis patients of both the IL-8 positive and negative group were not treated or were using only local therapy. Only five patients were treated with systemic steroids at the time of blood collection (three IL-8 positive and two negative respectively).

We observed more female patients $(n=43)$ than male patients $(n=18)$ with intermediate uveitis, but no relation was found between IL-8 in serum and sex of patients (data not shown). Furthermore no relation was observed between IL- 8 and age. Most patients were in the age group between 20 and $40(n=48)$, five were younger than 20 years of age, and 18 were older than 40 .

Follow up of the intermediate uveitis patients of whom the first were seen in 1981 ranged from 0.5 to 15 years with a mean of 4.5 years. Twenty of the 27 IL- 8 positive patients had a chronic disease with exacerbations. At the end of this study a relation with systemic disease was found in 10 out of 27 IL- 8 positive patients and in three out of 34 IL- 8 negative patients. Four patients had developed multiple sclerosis, of whom two had active uveitis with a high IL-8 level in serum at entry in the study

Table 3 IL-8 and disease activity in intermediate uveitis

\begin{tabular}{lccl}
\hline & IL-8 pos* & IL-8 neg & \\
& $(27)$ & $(34)$ & p Value \\
\hline Active & 23 & 9 & $<0.001$ \\
Smouldering & 4 & 25 & \\
\hline
\end{tabular}

${ }^{\star}$ IL-8 level above $20 \mathrm{pg} / \mathrm{ml}$.

Statistical analysis was performed using the $\chi^{2}$ test. and two had smouldering disease with low IL-8 levels. The time interval between developing multiple sclerosis after entry into our study ranged between 1 and 10 years with a mean of 4 years. Three patients are still suspected of having multiple sclerosis; all three had active uveitis with high IL-8 levels. Two of these three patients had a relative with multiple sclerosis. One patient with a high serum IL-8 level and active disease at entry in the study was diagnosed as having sarcoidosis 2 years later. Later follow up revealed that five patients had developed angiotensin converting enzyme levels above normal limits and were suspected for having sarcoidosis, four had active disease with high IL-8 levels, and one had smoldering disease with a low IL-8 level.

\section{Discussion}

In this report we show that patients with active intermediate uveitis of unknown origin have elevated serum IL-8 levels. The presence of circulating IL- 8 in active intermediate uveitis may suggest a systemic involvement in this type of inflammatory eye disease or at least suggest an immune pathogenesis of this disease. Whether this increased IL- 8 merely reflects an acute phase reaction in these patients, was investigated by measuring circulating CRP. CRP is an acute phase protein that is produced and secreted by hepatocytes in response to a wide variety of infections, inflammatory conditions, and tissue damage. Normally, the acute phase response lasts only a few days; however, in cases of chronic or recurring inflammation CRP can be elevated for prolonged periods. In this study we did not detect raised CRP levels in the patients with intermediate uveitis whether they had active or smoldering disease, which indicates that the increased IL-8 is not associated with an acute phase reaction.

IL-8 is a cytokine which attracts neutrophil granulocytes and $\mathrm{T}$ lymphocytes and is also involved in angiogenesis. ${ }^{11} 18 \mathrm{IL}-8$ is produced by leucocytes such as monocytes and macrophages but also by a variety of other cells such as fibroblasts, endothelial cells, and within the eye by retinal pigment epithelial cells and is triggered by bacterial endotoxins, tumour necrosis factor, and IL-1. ${ }^{19}{ }^{20}$ High levels of circulating IL-8 have been detected in various systemic diseases such as sarcoidosis ${ }^{13}$ and ulcerative colitis. ${ }^{14}$ Raised IL-8 serum levels were found in $81 \%$ of patients with chronic sarcoidosis. The serum IL-8 concentration was found to be higher in patients with active sarcoidosis than in patients with inactive sarcoidosis and all patients with active sarcoidosis had a serum IL-8 concentration greater than the normal range. ${ }^{13}$ The occurrence of eye disease was not reported in the latter study. Raised IL-8 levels were also reported in the serum of patients with eye diseases which are related to a systemic disease including proliferative diabetic retinopathy and HLA-B27 associated anterior uveitis. ${ }^{15}$

The source of circulating IL- 8 in active intermediate uveitis is not clear. It seems unlikely that cells in the inflamed peripheral retina and vitreous would produce such large 
amounts of IL- 8 that would lead to detectable levels in the blood compartment. In other uveitis entities we only observed significantly elevated IL-8 levels when systemic underlying disease was present (HLA-B27 acute anterior uveitis or sarcoidosis). This strongly suggests that another source than the eye is responsible for raised IL- 8 in active intermediate uveitis. Further evidence for systemic involvement in intermediate uveitis comes from studies in which increased levels of other immune markers such as soluble ICAM-1 were reported. ${ }^{21}$ More recently, Bora and colleagues found elevated serum levels of a $36 \mathrm{kDa}$ protein (nup36) in patients with active pars planitis that may be a marker for the disease. ${ }^{22}$

Twenty of the 27 IL- 8 positive patients had a chronic disease with exacerbations. The course and prognosis of intermediate uveitis has been reported to be variable: $10 \%$ of the patients have a self limiting disease with gradual amelioration without exacerbations, 59\% have a prolonged course without exacerbations; and $31 \%$ have a chronic smoldering disease with one or more subacute exacerbations and incomplete remissions. Those with moderately severe or severe disease are more apt to be in this final subgroup. ${ }^{3}$

Although intermediate uveitis may exist as an isolated idiopathic disorder, a number of underlying systemic diseases are associated with the disease, the most frequent include multiple sclerosis and sarcoidosis. An association between intermediate uveitis and HLADR15 has been reported. ${ }^{23}$ In HLA-DR15 positive intermediate uveitis patients about $50 \%$ had systemic findings of another HLADR15 related disorder (multiple sclerosis, optic neuritis, or narcolepsy). We have not yet performed HLA typing in the group of intermediate uveitis patients and can therefore not confirm these observations. In our study we excluded patients with a known systemic disease at entry. During follow up, however, four patients had developed multiple sclerosis, three patients were suspected for having multiple sclerosis, one patient developed sarcoidosis, and five had a presumed diagnosis of sarcoidosis. Since intermediate uveitis tends to be a disease of young adulthood, it is possible that at the time of diagnosis of the ocular condition, clinical and laboratory variables for these latter associated diseases may not yet be evident. In this study in $37 \%$ of the IL-8 positive intermediate uveitis patients an associated systemic disease was found 1-10 years later.
Larger numbers and longer follow up may provide definitive evidence as to whether a high IL-8 level is a marker of an increased likelihood of developing systemic disease in patients with intermediate uveitis.

1 Nussenblatt RB, Whitcup SM, Palestine AG. Uveitis: fundamentals and clinical practice. $2 \mathrm{~d}$ ed. Chapter 20: Intermediate uveitis. St Louis: Mosby, 1996:279-88.

2 Malinowski SM, Folk JC, Pulido JS. Pars planitis. Curr Opin Ophthalmol 1994;5:72-82.

3 Smith RE, Godfrey WA, Kimura SJ. Chronic cyclitis. I. Course and visual prognosis. Trans Am Acad Opthalmol Otolaryngol 1973;77:760-8.

4 Henderly DE, Genstler AJ, Rao NA, et al. Pars planitis. Trans Ophthalmol Soc UK 1986;105:227-32.

5 Zierhut M, Foster CS. Multiple sclerosis, sarcoidosis and other diseases in patients with pars planitis. Dev Ophthalmol 1992;23:41-7.

6 Malinowski SM, Pulido JS, Folk JC. Long-term visual outcome and complications associated with pars planitis. $O p h$ thalmology 1993;100:818-25.

7 Bamford CR, Ganley JP, Sibley WA, et al. Uveitis, perivenous sheating and multiple sclerosis. Neurology 1978 28:119-24.

8 Steel DM, Whitehead AS. The major acute phase reactants: C-reactive protein, serum amyloid $\mathrm{P}$ component and serum amyloid A protein. Immunol Today 1994;15:81-8.

$9 \mathrm{Kolb}-$ Bachofen V. A review on the biological properties of C-reactive protein. Immunobiol 1991;183:133-45.

10 Kilpatrick JM, Volanakis JE. Molecular genetics, structure and function of C-reactive protein. Immunol Res 1991;10 43-53.

11 Baggiolini M, Walz A, Kunkel SL. Neutrophil-activating peptide-1/interleukin 8 , a novel cytokine that activates neutrophils. F Clin Invest 1989;84:1045-9.

12 Hack CE, Hart M, Strack van Schijndel RJM, et al. Interleukin-8 in sepsis: relation to shock and inflammatory mediators. Infect Immun 1992;60:2835-42.

13 Yokoyama T, Kanda T, Kobayashi I, et al. Serum levels of interleukin-8 as a marker of disease activity in patients with chronic sarcoidosis. 7 Med 1995;26:209-19.

14 Mahida YR, Ceska M, Kurlak L, et al. Enhanced synthesis of neutrophil-activating peptide-1/interleukin- 8 in active ulcerative colitis. Clin Sci 1992;82:273-5.

15 de Boer JH, Hack CE, Verhoeven AJ, et al. Chemoattractant and neutrophil degranulation activities related to interleukin-8 in vitreous fluid in uveitis and vitreoretinal disorders. Invest Ophthalmol Vis Sci 1993;34:3376-85.

16 Bloch-Michel E, Nussenblatt RB. International Uveitis Study Group recommendations for the evaluation of intraocular inflammatory disease. Am f Ophthalmol 1987; intraocular

17 Whicher JT, Dieppe PA. Acute phase proteins. Clin Immunol Allergy 1985;5:425-46.

8 Strieter RM, Kunkel SL, Elner VM, et al. Interleukin-8: a corneal factor that induces neo-vascularization. $A m$ f Pathol 1992;141:1279-84.

19 Leonard EJ, Yoshima T. Neutrophil attractant/activation protein-1 (NAP-1 [Inter leukin-8]). Am $\mathcal{F}$ Respir Cell Mol Biol 1990;2:479-86.

20 Elner VM, Strieter RM, Elner SG, et al. Neutrophil chemotactic factor (IL-8) gene expression by cytokine-treated retinal pigment epithelial cells. Am f Pathol 1990;136:74550.

21 Arocker-Mettinger E, Steurer-Georgiew I, Steurer M, et al. Circulating ICAM-1 levels in serum of uveitis patients. Curr Eye Res 1992;11 (suppl): 161-6.

22 Bora NS, Bora PS, Kaplan HJ. Identification, quantitation, and purification of a $36 \mathrm{kDa}$ circulating protein associated with active pars planitis. Invest Ophthalmol Vis Sci 1996;37: 1870-6.

23 Tang WM, Pulido JS, Eckels DD, et al. The association of HLA-DR15 and intermediate uveitis. Am $\mathcal{f}$ Ophthalmol 1997;123:70-5. 